\title{
Genetic Basis for Variation of Metalloproteinase-Associated Biochemical Activity in Venom of the Mojave Rattlesnake (Crotalus scutulatus scutulatus)
}

\author{
Ruben K. Dagda, ${ }^{1}$ Sardar Gasanov, ${ }^{2}$ Ysidro De La OIII, ${ }^{3}$ Eppie D. Rael, ${ }^{3}$ and Carl S. Lieb ${ }^{3}$ \\ ${ }^{1}$ Pharmacology Department, University of Nevada School of Medicine, Manville Building 19A, Reno, NV 89557, USA \\ ${ }^{2}$ Science Department, Tashkent Ulugbek International School, 5-A J. Shoshiy Street, 100100 Tashkent, Uzbekistan \\ ${ }^{3}$ Department of Biological Sciences, University of Texas at El Paso, 500 West University Avenue, El Paso, TX 79968, USA
}

Correspondence should be addressed to Ruben K. Dagda; rdagda@medicine.nevada.edu

Received 26 April 2013; Accepted 25 June 2013

Academic Editor: R. J. Linhardt

Copyright (C) 2013 Ruben K. Dagda et al. This is an open access article distributed under the Creative Commons Attribution License, which permits unrestricted use, distribution, and reproduction in any medium, provided the original work is properly cited.

\begin{abstract}
The metalloproteinase composition and biochemical profiles of rattlesnake venom can be highly variable among rattlesnakes of the same species. We have previously shown that the neurotoxic properties of the Mojave rattlesnake (Crotalus scutulatus scutulatus) are associated with the presence of the Mojave toxin A subunit suggesting the existence of a genetic basis for rattlesnake venom composition. In this report, we hypothesized the existence of a genetic basis for intraspecies variation in metalloproteinaseassociated biochemical properties of rattlesnake venom of the Mojave rattlesnake. To address this question, we PCR-amplified and compared the genomic DNA nucleotide sequences that code for the mature metalloproteinase domain of fourteen Mojave rattlesnakes captured from different geographical locations across the southwest region of the United States. In addition, the venoms from the same rattlesnakes were tested for their ability to hydrolyze fibrinogen, fibrin, casein, and hide powder azure and for induction of hemorrhage in mice. Overall, based on genomic sequencing and biochemical data, we classified Mojave rattlesnake venom into four distinct groups of metalloproteinases. These findings indicate that differences in nucleotide sequences encoding the mature proteinase domain and noncoding regions contribute to differences in venom metalloproteinase activities among rattlesnakes of the same species.
\end{abstract}

\section{Introduction}

Rattlesnake venom metalloproteinases are zinc-dependent enzymes that hydrolyze fibrin and fibrinogen, inactivate complement proteins, and promote hemorrhage in vivo [14]. In addition, rattlesnake venom metalloproteinases show differences in substrate specificity, proteolytic activity, molecular weight, and composition of structural domains $[5,6]$.

Rattlesnake venom metalloproteinases are members of the Reprolysin superfamily of metalloproteinases [7]. These venom enzymes have been subcategorized into four classes (P-I to P-IV) based on the differences in structural domains, molecular weight, and biochemical properties $[7,8]$. The $\mathrm{P}$ I group of rattlesnake venom metalloproteinases contains a proteinase domain, whereas the P-II group contains an additional disintegrin-like domain. The P-III group has an additional disintegrin-like domain and cysteine-rich sequence, whereas the P-IV group has an additional lectin-like sequence [8].

The P-II and P-III classes of rattlesnake venom metalloproteinases are one- to twofold more potent at inducing hemorrhage than the P-I metalloproteinases [8-11]. This observation suggests that the disintegrin-like and cysteinerich domains play a critical role in inducing hemorrhage. In addition, the P-I metalloproteinases can be further divided into two subclasses, P-IA and P-IB. The P-IA subclass exhibits strong hemorrhagic activity, whereas the P-IB subclass has little or no hemorrhagic activity [12]. This observation implies that the P-IA metalloproteinases have certain structural features that are distinct from the disintegrin and cysteinerich domains for inducing hemorrhage [13].

Rattlesnake venoms differ significantly in metalloproteinase composition $[6,14-16]$. We have shown previously that rattlesnakes of the same species show differences 
in metalloproteinase composition and metalloproteinaseassociated biochemical activities $[4,6,14]$. Various posttranslational modifications of metalloproteinases may contribute to differences in biochemical properties of rattlesnake venom [17]. For instance, the venom of the Mojave rattlesnake shows differences in hemorrhage induction and proteolytic activities, which are associated with the geographic location where the rattlesnake was captured. Indeed, Mojave rattlesnakes from Texas either lack or contain modest hemorrhagic activity, while Mojave rattlesnakes from central Arizona contain high hemorrhagic activity and to the same extent as venom from Western Diamondback (C. atrox) and Northern Blacktailed (C. m. molossus) rattlesnakes [16]. In further support of this observation, we have previously reported significant intraspecies differences in the neurotoxic properties of rattlesnake venom of the Mojave rattlesnake $(C$. s. scutulatus) and Southern Pacific rattlesnake (C. helleri). These data suggest that both genetics and environmental factors contribute to intraspecies variation in rattlesnake venom composition and their biochemical properties $[18,19]$. A recent proteomics study of rattlesnake venom derived from twenty-one Mojave rattlesnakes showed an inverse relationship in the content of the Mojave toxin compared to snake venom metalloproteinase content which also correlated with geographical distribution of rattlesnakes [20].

In this study, we hypothesized that a genetic basis for intra-species biochemical variation of metalloproteinase activity exists in C. s. scutulatus. To address this question, we sequenced the proteinase domains of the metalloproteinase genes in fourteen Mojave rattlesnakes captured from different geographic locations of the southwest region of the United States. We did a battery of biochemical tests that evaluated metalloproteinase-associated proteolytic activity, nonmetalloproteinase proteolytic activity (esterases and nonzinc dependent serine proteases), and hemorrhagic activity. In summary, we categorized rattlesnake venom into four different groups based on their genomic DNA sequence homology and biochemical properties. Interestingly, the metalloproteinase gene of rattlesnake venom that lacked hemorrhagic activity contains a larger intronic region located within the proteinase domain and showed lower homology for the coding regions of the proteinase domain compared to the other groups of rattlesnake venom metalloproteinases. These observations suggest that differences in the noncoding region and single nucleotide polymorphisms located in the mature proteinase domain contribute to intra-species diversity in rattlesnake venom metalloproteinase composition.

\section{Materials and Methods}

2.1. Study Sample. Mojave rattlesnake venoms were obtained from snakes captured in Arizona, New Mexico, and Texas in the United States. The geographical information of the rattlesnakes is shown in Table 1.

2.2. DNA Extraction. Blood was withdrawn from the caudal vein of the rattlesnake and transferred to a tube containing sodium citrate to prevent coagulation. DNA was extracted
TABLE 1: Geographical data of Mojave rattlesnakes captured for this study.

\begin{tabular}{lc}
\hline Snake & Locality \\
\hline Css28 & El Paso County, TX, USA \\
Css31 & Offspring of Css28 \\
Css36 & Offspring of Css28 \\
Css61 & Hudspeth County, TX, USA \\
Css62 & Hudspeth County, TX, USA \\
Css64 & Hidalgo County, NM, USA \\
Css65 & Cochise County, AZ, USA \\
Css66 & Cochise County, AZ, USA \\
Css67 & Maricopa County, AZ, USA \\
Css68 & Maricopa County, AZ, USA \\
Css69 & Maricopa County, AZ, USA \\
Css71 & Maricopa County, AZ, USA \\
Css74 & Maricopa County, AZ, USA \\
Css75 & Maricopa County, AZ, USA \\
\hline
\end{tabular}

from whole blood using the DNA Zol reagent, a guanidine detergent (Molecular Research Center, Inc., Cincinnati, $\mathrm{OH}$, USA). After precipitation with isopropanol and washing with ethanol, the DNA was stored in $0.01 \mathrm{M}$ Tris-EDTA (TE) buffer. Quantification of DNA was done at OD260 $\mathrm{nm}$.

2.3. Oligonucleotides. Primers for amplifying the metalloproteinase genes were designed according to published cDNA sequences for snake venom metalloproteinases $[7,21,22]$. Primers were designed to amplify the mature proteinase domain by annealing to conserved regions within the zymogen and the spacer/disintegrin regions as verified through BLAST (National Center for Biotechnology). The primer pair, amplification sites, and corresponding rattlesnake venom metalloproteinase sequences are shown in Table 2.

2.4. PCR. DNA amplification was done using buffer C PCR kit (Invitrogen, Carlsbad, CA, USA). DNA (1-2 $\mu \mathrm{g})$, primers $(1 \mu \mathrm{M})$, Taq DNA polymerase (2.5 units), dNTP mix ( $1 \mathrm{mM})$, and $\mathrm{MgCl}_{2}(2.5 \mathrm{mM})$ were mixed in a total volume of $50 \mu \mathrm{L}$. PCR was done using the touchdown PCR method. In brief, the first seven cycles of PCR were done at a heat denaturation step of $95^{\circ} \mathrm{C}$ for $25 \mathrm{sec}$, an annealing step at $55^{\circ} \mathrm{C}$ for $30 \mathrm{sec}$, and an extension step at $72^{\circ} \mathrm{C}$ for $1 \mathrm{~min}$, which were followed by 32 cycles of PCR at a heat denaturation step at $95^{\circ} \mathrm{C}$ for $25 \mathrm{sec}$, an annealing step at $55^{\circ} \mathrm{C}$ for $30 \mathrm{sec}$, and an extension step at $45^{\circ} \mathrm{C}$ for $1 \mathrm{~min}$. The analysis of the amplified products was done by subjecting DNA products to electrophoresis in $1 \%$ agarose gels soaked in TAE (Tris-acetic acid-EDTA) buffer and visualizing with ethidium bromide when exposed in a UV box. A 100 base pair ladder (Amersham Pharmacia Biotech Inc.) was used to determine the size of products.

2.5. Cloning. PCR products were purified from the gel using the QIAquick Gel Extraction Kit (QIAGEN Inc., Valencia, CA, USA) and ligated into the pCR2.1 plasmid using the Original TA Cloning kit (Invitrogen, Carlsbad, CA, USA). The E. 
TABLE 2: Primers used for amplifying metalloproteinase sequences from Crotalus s. scutulatus. Genomic DNA and sequence comparison to other snake venom metalloproteinases (MP). Nonconserved nucleotides are indicated in bold.

\begin{tabular}{|c|c|c|}
\hline Primer & Annealing site & Corresponding sequence \\
\hline \multirow[t]{6}{*}{ MP (sense) } & & GCCCCCAAAATGTGTGGGGTAAC \\
\hline & Atrolysinb (558-580) & GCCСCCAAAATGTGTGGGGTAAC \\
\hline & Artolysinc (567-589) & GCCСССAAAATGTGTGGGGTAAC \\
\hline & Atrolysind (488-510) & GCССССАAAATGTGTGGGGTAAC \\
\hline & Atrolysine (548-570) & GCCСССAAAATGTGTGGGGTAAC \\
\hline & Catrocollastatin (537-559) & GCССТCAAAATGTGTGGGGTAAC \\
\hline \multirow[t]{7}{*}{ MP (antisense) } & & TTCTTCTCCCGCCTCCAAAAGTTC \\
\hline & Atrolysina (1329-1306) & ТTСТТСТСССАССТССААААGTТС \\
\hline & Atrolysinb (1328-1305) & TTCTTCTCCCGCCTCCAAAAGTTC \\
\hline & Atrolysinc (1327-1304) & TTCTTCTCCCGCCTCCAAAAGTTC \\
\hline & Atrolysind (1248-1225) & TTCTTCTCCCGCCTCCAAAAGTTC \\
\hline & Atrolysine (1308-1285) & TTCTATTCCCGCCTCCAAAAGTTC \\
\hline & Catrocollastatin (1297-1274) & TTCTTCTCCGGCTTCCAAAAGTTC \\
\hline
\end{tabular}

coli strain TOP10F' competent cells were then transformed with DNA ligation reactions. Cells were grown overnight at $37^{\circ} \mathrm{C}$ on TSB plates containing kanamycin $(100 \mu \mathrm{g} / \mathrm{mL})$, IPTG $(0.1 \mathrm{M})$, and X-Gal $(25 \mu \mathrm{g} / 1.25 \mathrm{~mL})$. White colonies were replated and grown overnight to ensure that the colonies were transformants. Positive clones were grown overnight at $37^{\circ} \mathrm{C}$ in $10 \mathrm{~mL}$ of TSB broth. Plasmids were isolated from the culture using the Wizard Plus Minipreps DNA Purification kit (Promega, madison, WI, USA) and examined for the presence of DNA inserts by EcoRI digestion.

2.6. DNA Sequencing. Plasmid DNA containing genomic DNA inserts was subjected to PCR using $7.5 \times 10^{-8} \mathrm{M}$ of M13 Forward (-29)/IRD700 and M13 Reverse/IRD800 dye-labeled primers (LI-COR, Lincoln, NE, USA) following the SequiTherm EXCEL II DNA Sequencing Kit-LC protocol for $66 \mathrm{~cm}$ gels (Epicentre Technologies, Madison, WI, USA). PCR products were electrophoresed in 3.6\% polyacrylamide gel in TBE (Tris-Base EDTA) buffer on a LI-COR LongReadIR 4200 DNA Sequencer at $2000 \mathrm{~V}$, $25.0 \mathrm{~mA}$, and $45.0 \mathrm{~W}$, at $45^{\circ} \mathrm{C}$. Each DNA sequence was confirmed, in triplicate tests, by purifying and sequencing DNA from different clones. The nucleotide sequences were analyzed using the Vector NTI Suite program (InforMax, Inc., North Bethesda, MD, USA), a registered trademark of Life Technologies (Grand Island, NY, USA). The nucleotide sequences were aligned to known sequences using the BLAST program in the FASTA format.

2.7. Slot Blot Assay of Rattlesnake Venom. In brief, $2 \mu \mathrm{L}$ of rattlesnake venom was spotted onto nitrocellulose strips, washed five times in PBS, blocked in 5\% goat serum, and incubated with metalloproteinase-specific antibodies AF5 and CAP-8, antibodies raised against anticomplement factors, and hemorrhagic metalloproteinases in C. s. scutulatus and C. atrox, respectively. The nitrocellulose strips were then washed extensively in PBS and incubated with secondary antibodies conjugated to horseradish peroxidase and developed using standard chromogenic reactions as previously described [3]. CAP-8 (a gift from Dr. John Perez, Texas A\&M University, College Station, TX, USA) is a mouse monoclonal antibody that recognizes hemorrhagic rattlesnake venom proteins from rattlesnake venoms of C. atrox. AF5 is a polyclonal antibody raised against hemorrhagic and complement inactivating metalloproteinases derived from C. s. scutulatus venom. Antibody specificities have been characterized in previous studies [23-25].

2.8. Fibrinogenolytic Activity. Fibrinogenolytic activity in rattlesnake venom was assessed by mixing $160 \mu \mathrm{L}$ of a $1 \mathrm{mg} / \mathrm{mL}$ solution of human fibrinogen ( $93 \%$ clottable protein), dissolved in $0.03 \mathrm{M}$ ammonium acetate ( $\mathrm{pH} 7.5$ ), with $16 \mu \mathrm{L}$ of rattlesnake venom $(1 \mathrm{mg} / \mathrm{mL})$ and $224 \mu \mathrm{L}$ of distilled water. Reaction mixtures were incubated at $37^{\circ} \mathrm{C}$ for $30 \mathrm{~min}$. The reaction was terminated by adding $5 \mu \mathrm{L}$ of $0.5 \mathrm{M}$ EDTA to $5 \mu \mathrm{L}$ aliquots of each sample.

For some experiments, the biochemical activity associated with $\mathrm{Zn}^{2+}$-dependent metalloproteinases was assessed by incubating the reaction mixtures with EDTA to chelate zinc. In brief, $50 \mu \mathrm{L}$ of rattlesnake venom $(1 \mathrm{mg} / \mathrm{mL})$ was incubated with $20 \mathrm{mM}$ EDTA for $1 \mathrm{hr}$ at $37^{\circ} \mathrm{C}$. The EDTA concentration in this mixture was two times higher in order to ensure the sequestration of $\mathrm{Zn}^{2+}$ and inactivation of metalloproteinases.

2.9. Fibrinolysis. Fibrinolytic activity was measured in fibrinagarose plates that were prepared by mixing $2.5 \mathrm{~mL}$ of $1 \%$ agar with a $1 \%$ fibrinogen suspension to $35 \mathrm{~mm}$ Petri dishes containing $0.1 \mathrm{~mL}$ thrombin ( 0.5 units) to induce fibrin formation. Ten $\mu \mathrm{L}$ of venom $(1 \mathrm{mg} / \mathrm{mL})$ was added to $3 \mathrm{~mm}$ wells in the agarose gels. Following incubation for $12 \mathrm{hr}$ at $37^{\circ} \mathrm{C}$ in a humidified chamber, the diameters of the lysis zones induced by rattlesnake venom were measured with a ruler. In EDTA inhibition reactions, samples of venom were preincubated with $20 \mathrm{mM}$ EDTA for $1 \mathrm{hr}$ at $37^{\circ} \mathrm{C}$. Standard 
deviations were determined from three separate repetitions of the procedure.

2.10. Caseinolysis. Caseinolytic activity was assayed in casein agar plates that were prepared by mixing agar with Protease Substrate Gel Tablets (Bio-Rad, Richmond, CA, USA) according to the manufacturer's protocol. Ten $\mu \mathrm{L}$ of each sample $(1 \mathrm{mg} / \mathrm{mL}$ venom in $0.1 \mathrm{M}$ Tris-HCl buffer, $\mathrm{pH}$ 8.2) was dispersed into $4 \mathrm{~mm}$ diameter wells that were punched in the gel following polymerization of agar containing casein. The gels were then incubated at $37^{\circ} \mathrm{C}$ in humidified chamber for $12 \mathrm{hr}$. The caseinolytic activity of rattlesnake venom samples was determined by measuring the diameter of the hydrolysis zones. For EDTA treated reactions, venom samples were preincubated with $20 \mathrm{mM}$ EDTA for $1 \mathrm{hr}$ at $37^{\circ} \mathrm{C}$.

2.11. Esterase Activity. Esterase activity was determined by adding $50 \mu \mathrm{L}$ of venom $(1 \mathrm{mg} / \mathrm{mL})$ to $300 \mu \mathrm{L}$ of either $0.01 \mathrm{M}$ $\mathrm{N}$-a-p-tosyl-L-arginine methyl ester (TAME) or $0.01 \mathrm{M} \mathrm{N}$-abezoyl-L-arginine ethyl ester (BAEE) dissolved in $2.55 \mathrm{~mL}$ of $0.1 \mathrm{M}$ Tris- $\mathrm{HCl}$ buffer, $\mathrm{pH} 8.2$, containing $0.05 \mathrm{M} \mathrm{CaCl}_{2}$ and incubating for $1 \mathrm{hr}$ at $37^{\circ} \mathrm{C}$. Reaction was stopped by addition of $0.1 \mathrm{~mL}$ glacial acetic acid. Absorbance was read at $247 \mathrm{~nm}$ for TAME and $253 \mathrm{~nm}$ for BAEE. The activity is reported as the change in absorbance per min per mg protein. Each reaction was done at least in triplicate.

2.12. Hide Powder Azure Hydrolysis. Hydrolysis of hide powder azure was determined by adding $50 \mu \mathrm{L}$ of venom $(1 \mathrm{mg} / \mathrm{mL})$ to $5 \mathrm{mg}$ of Remazol Brilliant Blue hide powder azure (Calbiochem Corp., La Jolla, CA, USA) suspended in $1.85 \mathrm{~mL}$ of $0.1 \mathrm{M}$ Tris- $\mathrm{HCl}, 0.05 \mathrm{M} \mathrm{CaCl}_{2}, \mathrm{pH}$ 8.2. Reaction was terminated, after an incubation period of $2.5 \mathrm{hr}$ at $37^{\circ} \mathrm{C}$, by addition of $0.1 \mathrm{~mL}$ glacial acetic acid. The samples were centrifuged in a Microfuge for $15 \mathrm{~min}$. For EDTA treated reactions, venom samples were preincubated with $20 \mathrm{mM}$ EDTA for $1 \mathrm{hr}$ at $37^{\circ} \mathrm{C}$. Optical absorbance of the supernatant was determined at $595 \mathrm{~nm}$. Specific activity is reported as the change in optical absorbance per min per mg protein. Each reaction was done at least in triplicate in three independent experiments.

2.13. Hemorrhagic Activity. Hemorrhagic activity of each rattlesnake venom sample was determined by injecting adult $\mathrm{BALB} / \mathrm{c}$ mice subcutaneously in the dorsal region with $100 \mu \mathrm{g}$ of venom dissolved in $100 \mu \mathrm{L}$ of $0.85 \% \mathrm{NaCl}$. Following cervical dislocation, mice were examined for hemorrhage $4 \mathrm{hr}$ after injection. Hemorrhagic activity is reported on a relative scale from zero to four, where four indicates high hemorrhagic activity (hemorrhagic area greater than $10 \mathrm{~mm}$ in diameter) and zero indicates no hemorrhagic activity.

\section{Results}

The enzymatic and hemorrhagic activities of fourteen C. $s$. scutulatus venoms are shown in Table 3. For comparative purposes, we also included data obtained from venoms from C. atrox (Call-1) and C. m. molossus (Cmm88) as positive controls for both proteolytic and hemorrhagic activities [26, 27]. We found that C. s. scutulatus venoms from Css28, Css31, and Css36 (collected from El Paso, TX, USA) showed potent hemorrhagic activity. Venom from three other C. $s$. scutulatus Css68, Css71, and Css74 collected in Maricopa County, AZ, USA, also induced hemorrhage to the same extent as venom derived from C. atrox. The other eight $C$. $s$. scutulatus venoms (collected from various geographic locations including Maricopa County, AZ, USA) did not cause hemorrhage (Table 3).

The presence of hemorrhagic toxins in venoms was also assessed by immunoblotting for hemorrhagic metalloproteinases (Table 3 ) using the slot blot method as previously published $[3,28]$. Both AF5 and CAP8 antibodies immunoreacted with venoms that contained high hemorrhagic activity, whereas CAP8, but not AF5, recognized venoms with weak hemorrhagic activity (Css71 and Css74).

Significant differences were observed in proteolytic activities of rattlesnake venoms, particularly in the ability to hydrolyze fibrin, casein, and hide powder azure (HPA). A strong correlation was observed in the ability of rattlesnake venom to induce hemorrhage with its ability to hydrolyze HPA, an observation previously reported [16]. However, while all venoms were capable of hydrolyzing fibrinogen, some venoms (Css61, Css62, and Css64) lacked caseinolytic, fibrinolytic, and hemorrhagic activities (Table 3).

The venoms were pretreated with EDTA to ensure that the proteolytic activities were associated with metalloproteinases. The venoms were then tested for their ability to hydrolyze the esterase substrates TAME and BAEE. As shown in Table 3, all rattlesnake venoms had activity for both TAME and BAEE. The ability of EDTA pretreated venom to cleave fibrinogen varied (Table 4). While EDTA completely inhibited the fibrinogenolytic activity of venom Css61, EDTA was only able to partially inhibit fibrinogenolytic activity in four other venoms while having no effect on the rest of the rattlesnake venoms. These observations show that nonmetalloproteinase enzymes like esterase or serine proteases $[29,30]$ are capable of cleaving fibrinogen and were present in most of the venoms. However, hydrolysis of casein, fibrin, and HPA was completely inhibited by EDTA suggesting that all of the venoms contained zinc-dependent metalloproteinases that hydrolyze casein and fibrin, including venoms that lacked hemorrhagic activity and those that were not recognized by either the AF5 or CAP8 antibodies. In summary, all the biochemical activities including caseinolysis, fibrinolysis, and cleavage of HPA were attributed to metalloproteinases (Table 4).

Based on the results shown in Table 3, we were able to classify the snake venoms into four groups. Venoms from Css28, Css31, Css36, and Css68 were categorized in group one (GP1) based on their high proteolytic activity on all substrates analyzed and their high hemorrhagic activity. The second group (GP2) included Css61, Css62, and Css64. These three venoms hydrolyzed only fibrinogen but were not active against fibrin, casein, or hide powder azure and lacked hemorrhagic activity. The third group (GP3) included Css65, Css66, Css67, Css69, and Css75, all of which were active on all protein substrates except high powder azure and did not cause hemorrhage. Finally, the fourth group (GP4) included 
TABLE 3: Antibody recognition: proteinase and hemorrhagic activities of the C. s. scutulatus venoms.

\begin{tabular}{|c|c|c|c|c|c|c|c|c|c|}
\hline Snake & $\begin{array}{l}\text { Fibrinogenolytic } \\
\text { activity (peptide } \\
\text { cleaved) }\end{array}$ & $\begin{array}{c}\text { Fibrinolytic } \\
\text { activity }(\mathrm{mm})\end{array}$ & $\begin{array}{l}\text { Caseinolytic } \\
\text { activity }(\mathrm{mm})\end{array}$ & $\begin{array}{l}\text { TAME }^{\mathrm{a}} \\
\text { hydrolysis } \\
\text { (units) }\end{array}$ & $\begin{array}{c}\text { BAEE }^{\mathrm{b}} \\
\text { hydrolysis (units) }\end{array}$ & $\begin{array}{c}\text { HP azure } \\
\text { hydrolysis (units) }\end{array}$ & $\begin{array}{l}\text { HEM }^{\mathrm{d}} \text { activity } \\
\text { (intensity) }\end{array}$ & AF5 & CAP8 \\
\hline Css 28 & $\alpha \beta$ & $11 \pm 0.3^{\mathrm{e}}$ & $9.0 \pm 0.3$ & $1.66 \pm 0.03$ & $1.131 \pm 0.02$ & $0.161 \pm 0.01$ & $4+$ & + & + \\
\hline Css 31 & $\alpha \beta$ & $8.0 \pm 0.2$ & $7.0 \pm 0.2$ & $1.40 \pm 0.02$ & $1.30 \pm 0.02$ & $0.211 \pm 0.01$ & $4+$ & + & + \\
\hline Css36 & $\alpha \beta$ & $6.0 \pm 0.2$ & $6.0 \pm 0.2$ & $1.15 \pm 0.01$ & $0.901 \pm 0.01$ & $0.171 \pm 0.01$ & $4+$ & + & + \\
\hline Css61 & $\alpha \beta$ & 0 & 0 & $1.72 \pm 0.02$ & $0.691 \pm 0.05$ & 0 & 0 & - & - \\
\hline Css62 & $\alpha \beta$ & 0 & 0 & $0.18 \pm 0.01$ & $0.441 \pm 0.03$ & 0 & 0 & - & - \\
\hline Css64 & $\alpha \beta$ & 0 & 0 & $0.931 \pm 0.02$ & $0.51 \pm 0.02$ & 0 & 0 & - & - \\
\hline Css65 & $\alpha \beta$ & $15 \pm 0.6$ & $3.5 \pm 0.1$ & $3.50 \pm 0.01$ & $\mathrm{ND}^{\mathrm{f}}$ & 0 & 0 & - & - \\
\hline Css66 & $\alpha \beta$ & $4.0 \pm 0.1$ & $6.01 \pm 0.3$ & $0.261 \pm 0.02$ & $0.481 \pm 0.03$ & 0 & 0 & - & - \\
\hline Css67 & $\alpha \beta$ & $5.0 \pm 0.2$ & $5.0 \pm 0.2$ & $0.161 \pm 0.01$ & $0.061 \pm 0.001$ & 0 & 0 & - & - \\
\hline Css68 & $\alpha \beta$ & $8.0 \pm 0.3$ & $11.0 \pm 0.4$ & $0.081 \pm 0.001$ & $0.081 \pm 0.001$ & $0.281 \pm 0.01$ & $4+$ & + & + \\
\hline Css69 & $\alpha \beta$ & $6.0 \pm 0.2$ & $4.0 \pm 0.1$ & $0.50 \pm 0.01$ & ND & 0 & 0 & - & - \\
\hline Css71 & $\alpha \beta$ & $6.0 \pm 0.2$ & $3.5 \pm 0.1$ & $0.301 \pm 0.01$ & $0.441 \pm 0.03$ & $0.061 \pm 0.02$ & $1+$ & - & + \\
\hline Css74 & $\alpha \beta$ & $6.0 \pm 0.2$ & $6.5 \pm 0.2$ & $0.221 \pm 0.02$ & $0.221 \pm 0.01$ & $0.061 \pm 0.01$ & $1+$ & - & + \\
\hline Css75 & $\alpha \beta$ & $8.0 \pm 0.3$ & $3.51 \pm 0.1$ & $0.321 \pm 0.03$ & $0.321 \pm 0.01$ & 0 & 0 & - & - \\
\hline Call-1 & $\alpha \beta$ & $18 \pm 0.5$ & $14.0 \pm 0.5$ & ND & ND & $0.5191 \pm 0.02$ & $4+$ & + & + \\
\hline Cmm88 & $\alpha \beta$ & $22 \pm 0.9$ & $16.0 \pm 0.6$ & $0.881 \pm 0.214$ & $0.871 \pm 0.32$ & $0.321 \pm 0.01$ & $4+$ & ND & ND \\
\hline
\end{tabular}

${ }^{\mathrm{a}} \mathrm{N}$-a-Tosyl-L-arginine methyl ester.

${ }^{\mathrm{b}} \mathrm{N}$-a-Benzoyl-L-arginine ethyl ester.

${ }^{\mathrm{c}}$ Hide powder azure.

${ }^{\mathrm{d}}$ Hemorrhagic.

${ }^{\mathrm{e}}$ Mean \pm the standard deviation based on three repetitions.

${ }^{\mathrm{f}}$ No data.

TABLE 4: Proteolytic activity inhibition of the C. s. scutulatus venoms by EDTA.

\begin{tabular}{lccc}
\hline Snake & $\begin{array}{c}\text { Inhibition of } \\
\text { fibrinogenolysis } \\
\text { (peptide cleaved) }\end{array}$ & $\begin{array}{c}\text { Inhibition of } \\
\text { caseinolysis and } \\
\text { fibrinolysis }\end{array}$ & $\begin{array}{c}\text { Inhibition of } \\
\text { hide powder } \\
\text { azure hydrolysis }\end{array}$ \\
\hline Css28 & $\beta$ & $+^{\mathrm{a}}$ & + \\
Css31 & $\beta$ & + & + \\
Css36 & $\alpha \beta$ & + & + \\
Css61 & None & NA & NA \\
Css62 & $\beta$ & $\mathrm{NA}$ & $\mathrm{NA}$ \\
Css64 & $\beta$ & $\mathrm{NA}$ & $\mathrm{NA}$ \\
Css65 & $\alpha \beta$ & + & $\mathrm{NA}$ \\
Css66 & $\alpha \beta$ & + & $\mathrm{NA}$ \\
Css67 & $\alpha \beta$ & + & $\mathrm{NA}$ \\
Css68 & $\beta$ & + & + \\
Css69 & $\alpha \beta$ & + & $\mathrm{NA}$ \\
Css71 & $\alpha \beta$ & + & + \\
Css74 & $\alpha \beta$ & + & + \\
Css75 & $\alpha \beta$ & + & $\mathrm{NA}$ \\
\hline
\end{tabular}

$a_{+}$indicates that the activity was inhibited $100 \%$ by EDTA.

${ }^{\mathrm{b}}$ Not applicable due to the lack of activity when not treated with EDTA (refer to Table 3).

Css71 and Css74. These venoms were active on all protein substrates but were weakly hemorrhagic (Table 3).
Genomic DNA sequences (four groups of genomic metalloproteinase DNA sequences, GP-I, GP-II, GP-III, and GP-IV, have been deposited in the GenBank under the accession numbers AF378673, AF378674, AF378675, and AF378676, resp.) were subsequently determined for the metalloproteinase genes. Using primers shown in Table 2, we were able to PCR-amplify 1,050 base pair metalloproteinase specific gene fragments for each of the 14 C. s. scutulatus rattlesnakes. The PCR-amplified nucleotide fragments were extracted from the agarose gels, subcloned into TOPO vectors, and sequenced (Figure 1). Following DNA sequence alignments using BLAST algorithm, each genomic DNA sample showed high homology to known metalloproteinase genes. The highest sequence identity belonged to the cDNAs of C. atrox Atrolysins [7] and Agkistrodon contortrix (copperhead) metalloproteinase genes [11,31-33]. One intron was identified to be present in all of the partial genomic metalloproteinase DNA sequences analyzed in the sample C. s. scutulatus population. This intron was located seven nucleotides (from the $5^{\prime}$ end) inside the $\mathrm{Zn}^{2+}$-binding site (base pair number 476, see Supplementary Figure 1 in supplementary material available online at http://dx.doi.org/10.1155/2013/251474). This intron had a classical consensus splice-site (GT donor and AG acceptor) sequence (Supplementary Figure 1).

All of the nucleotide sequences contained similar structural domains including a conserved "cysteine switch" sequence [34] located within the zymogen region, an $\mathrm{N}$ terminal region of the proteinase domain, a $\mathrm{Zn}^{2+}$-binding 
GP1

GP2

GP3

GP4

66

¿"Cysteine switch" sequence (PKMCGVT)

GGCCCCAAAATGTGTGGGGTAACCAGACTACTTGGGAATCAGATGAGCCCATCAAAAAGCTTCTC GCCCCTAAAATGTGTGGGGTAACCAGACTACTTGGGAATCATATGAGCCCATCAAAAAGCTTCTC GCCCCCAAAATGTGTGGGGTAACCAGACTACTTGGGAATCAGATGAGCCCATCAAAAAGCTTCTC GCCCCCAAAATGTGTGGGGTAACCAGACTACTTGGGAATCAGATGAGCCCATCAAAAAGCTTCTC

130

GP1

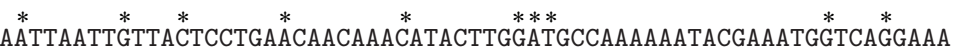

GP2

GP3

GP4 ATTTAATTCTTAATCCTGACCAACAAAGATACTTGGATGCCAAAAAATACGAAATGTTCATGAAA AGTTAATTGTTACTCCTGAACAACAAACATACTTGGATGCCAAAAAATACGAAATGGTCAGGAAA AGTTAATTGTTACTCCTGAACAACAAACATACTTGCGAGCCAAAAAATACGAAATGGTCAGGAAA $\uparrow \mathrm{N}$-terminal region of mature protein [31]

131

GP1

GP2

GP3

GP4

196

260

GP1

TTCAATATTCGTGTACCATTCACTGGCCTACAAATTTGGTCCAATÄGAGATTTCATTAAATGTGC TTCAATATTGATGTCTCATTCACTGGCCTACAAATTTGGTCCAATCGGGATTTCATTAAATGTGC TTCAATATTCGTGTACCATTCACTGGCCTACAAATTTGGTCCAATAGAGATTTCATTAAATGTGC TTCAATATTCGTGTACCATTCACTGGCCTACAAATTTGGTCCAATAGAGATTTCATTAAATGTGC

261

325

GP1

AGTCAGCAGTAÄTGTTACTTTGGACTTATTTGGAGAATGGAGAGAAGACAGTCTTGCTGAATCG

GP2 AGTCATCAGTAAATGTTACTTTGGACTTATTTGGAGAATGGAGAGAAGACAGTCTTGCTGAATCG GP3 AGTCAGCAGTAACTGTTACTTTGGACTTATTTGGAGAATGGAGAGAAGAAAGTCTTGCTGAATCG GP4 AGTCAGCAGTAACTGTTACTTTGGACTTATTTGGAGAATGGAGAGAAGAAAGTCTTGCTGAATCG

326

390

GP1

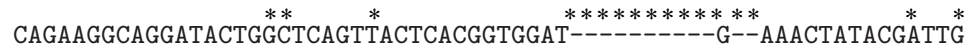
CAGAAGGCAGGATACTGCGTCAGTCACTCACGGTGGATAACTTTGGAGGAAAAACTATACGATTT CAGAAGGCAGGATACTGGCTCAGTTACTCACGGTGGAT-----------G--AAACTATACCATTG CAGAAGGCAGGATACTGGCTCAGTTACTCACGGTGGAT-----------G--AAACTATACCATTG

391 455

GP1 GGCAGCATGTGCCACCCGAAGCTTTCTGCAGGAATTGTTCAGGTTCATAGCACATTACATTTATT GP2 GGCAGCACGTGCAACCCGAGGTATTCTGCAGGAATTGTTAAGGATCATAGCCCAATACATCTCAC GP3 GGCAGCATGTGCCACCGGAAGCTTTCTGCAGGAATTGTTCAGGATCATAGCACAATACATTTTAT GP4 GGCAGCATGTGCCACCGGAAGCTTTCTGCAGGAATTGTTCAGGATCATAGCACAATACATTTTAT

(a)

Figure 1: Continued. 
GP1

GP2

GP3

GP4

531

$\stackrel{* *}{*} \stackrel{*}{*} \stackrel{*}{*} \underset{*}{*} \stackrel{* *}{*} \stackrel{*}{*} \stackrel{*}{*} \quad \stackrel{*}{*} \stackrel{* * *}{* *}$ TGGTGGGAATTACTATGGCCCATGAGCGTGATCTGGAACCTAAGGCACGTGGGCCATAGGTGGCACATCGTCCAG GGTTGGAAATTACAATGGCCCATGAGCGTGATGGCGAACCTA-GGCACGTGTGCCATAGGTGGCACACCATCGCC GGTTGGAAATTACAATGGCCCATGAGCGTGATGGCGAACCTA-GGCACGTGTGCCATAGGTGGCACACCATCGCC $\uparrow \mathrm{Zn}^{2+}$-binding motif start
GP1

GP2

GP3

GP4

613

$* * * * * * * * * * * * * * * * * * * * * * * * * * * * * * * * * * * * * * * * * * * * * * * * * * * * * * * * * * * * * * * * *$ CAA-TTTCAACTTCTCGC--

GP1

GP2

GP3

GP4

695

776

GP1

GP2

GP3

GP4

777

858

GP1

GP2

GP3

GP4

$\stackrel{*}{*} \stackrel{*}{*} \stackrel{*}{\text { CACACCCACCC-AATTGGCCAAACCTACCCGA-TGGGCTACGCCTACCTGGTACTCAACGATACATAAGTGGGTTTTGGTTT }} \stackrel{*}{*}$ CAC-CCCCCCCCAATTGGCCAACCCCCCCCGAGTGGTACGCCTC--CCTGGTACCCAACGATACATAAGTGGGTTTTGGTTT CACACCCACCC-AATTGGCCAAACCTACCCG-GTGGGCTACACCTACCTGGTACCCAACGATACATAAGTGGGTTTTGGTTT CACACCCACCC-AATTGGCCAAACCTACCCG-GTGGGCTACACCTACCTGGTACCCAACGATACATAAGTGGGTTTTGGTTT

934

GP1 TGACAGAGATTCCTGTACTTGCGGTGCTAACTCATGCATTATGGCTTCTGTTCTAAGTGATCAAT GP2 TGACAGAAATTCCTGTACTTGCGGTGCTAACTCATGCATTATGGCTTCTGTTCTAAGCGATCAAT GP3 TGACAGAAATTCCTGTACTTGGGGTGCTAACTCATGCATTATGGCTTCTGTTCTAAGTGATCAAT GP4 TGACAGAAATTCCTGTACTTGCGGTGCTAACTCATGCATTATGGCTTCTGTTCTAAGTGATCAAT $\uparrow \mathrm{Zn}^{2+}$-binding motif end

(b)

FIgURe 1: Continued.
GP3

GP4
859

933 GCAGTTTGGGCACTCAGTCTCTAAAAGGTTCACCATCACTGGGCAATGAGTGGGTCATAATCTGGGCATTAATCA
612

694 
GP1

GP2

GP3

GP4

\begin{tabular}{|c|c|}
\hline & 1064 \\
\hline GP1 & CAATGCATTCTCAATGAACC \\
\hline GP2 & CAATGCATTCTCAATGACCC \\
\hline GP3 & CAATGCATTCTCAATGAACC \\
\hline \multirow[t]{2}{*}{ GP4 } & CAATGCATTCTCAATGAACC \\
\hline & 1129 \\
\hline GP1 & TTTGGAGGCGGGAGAAGAA \\
\hline GP2 & TTTGGAGGCGGGAGAAGAA \\
\hline GP3 & TTTGGAGGCGGGAGAAGAA \\
\hline GP4 & TTTGGAGGCGGGAGAAGA \\
\hline
\end{tabular}

CTTCCAATTCATTCAGCAATTA* $\stackrel{*}{*}$ AGTAAGGTGGAACATAGGAGGTATCTTATTAATCATÄCCCCA CTTCCAATTCATTCAGCAATTGTAGTAAGGTGGAACATAGGAGGTATCTTATTAATCATCCCCCA CTTCCAATTCATTCAGCAATTATAGTAAGGTGGAACATAGGAGGTATCTTATTAATCATACCCCA CTTCCAATTCATTCAGCAATTATAGTAAGGTGGAACATAGGAGGTATCTTATTAATCATACCCCA

(c)

FIGURE 1: Alignment of the four groups (GP1, GP2, GP3, and GP4) of C. s. scutulatus metalloproteinase genomic DNA sequences. Assignment of exon and intron (underlined) regions was made by comparing metalloproteinase cDNA sequences from C. atrox [7] and A. contortrix $[12,30]$ with metalloproteinase genomic DNA sequences from C. s. scutulatus obtained in this study. Differences among the DNA sequences are denoted by an asterisk $\left({ }^{*}\right)$.

TABLE 5: DNA sequence homology for different structural domains of the four groups of C. s. scutulatus metalloproteinase genes.

\begin{tabular}{lcccc}
\hline $\begin{array}{l}\text { Group number } \\
\text { domain }\end{array}$ & Total & $\begin{array}{c}\text { Proteinase } \\
\text { domain }\end{array}$ & Intron & $\begin{array}{c}\text { Zinc-binding } \\
\text { domain }\end{array}$ \\
\hline GP1 versus GP2 & $84.5 \%$ & $89 \%$ & $67.3 \%$ & $100 \%$ \\
GP1 versus GP3 & $98 \%$ & $99 \%$ & $94.7 \%$ & $100 \%$ \\
GP1 versus GP4 & $98 \%$ & $99 \%$ & $96 \%$ & $100 \%$ \\
GP2 versus GP3 & $83 \%$ & $89 \%$ & $67 \%$ & $100 \%$ \\
GP2 versus GP4 & $83 \%$ & $90 \%$ & $70 \%$ & $100 \%$ \\
GP3 versus GP4 & $99 \%$ & $99 \%$ & $100 \%$ & $100 \%$ \\
\hline
\end{tabular}

domain, and a spacer domain located between the proteinase and disintegrin domains. The homology among the genomic DNA metalloproteinase sequences was greater than $95 \%$ for the zymogen region (nucleotides 1-82), 100\% for the zincbinding domain (Table 5), and greater than $98 \%$ for the spacer region (nucleotides 1084 to 1147). The DNA sequence homology for the proteinase domain across all 14 C. s. scutulatus genomic metalloproteinase genes, excluding the intron (nucleotides 83 to 474 and 909 to 1083), was close to $89 \%$, and the sequence identity for the entire gene was at least $84.5 \%$ homologous among the four groups of metalloproteinase genes (Table 5). However, the sequence homology within the intron region among the fourteen snakes (nucleotides 476 to 908$)$ was highly variable ( $67 \%$ homology for some rattlesnakes, Table 5) suggesting that differences in the splicing of introns and fusion of exons among the four different groups of rattlesnake venoms may contribute to variations in metalloproteinase activities as shown in Table 3.
A thorough analysis of the DNA nucleotide sequence showed that the fourteen metalloproteinase genomic DNA sequences exhibited a perfect correlation with the biochemical classification of the rattlesnake venoms. The genomic DNA sequences from Css28, Css31, Css36, and Css68 were classified in group one (GP1), whereas genomic DNA sequences belonging to Css61, Css62, and Css64 were classified into group two (GP2). The genomic DNA sequences belonging to Css65, Css66, Css67, Css69, and Css75 were categorized into group three (GP3), whereas genomic DNA sequences belonging to Css71 and Css74 were classified in group four (GP4) (Figure 1). This classification is identical to the classification that was based on the biochemical profiles of the fourteen rattlesnake venoms pointing to the existence of a genetic basis that gives rise to intra-species variation in metalloproteinase-associated activities in C. s. scutulatus. Interestingly, the genomic DNA sequence belonging to GP2 differed the most compared to the other three groups in that it contained a longer intron sequence (Table 5).

\section{Discussion}

C. s. scutulatus is an excellent rattlesnake species for studying intra-species variations in rattlesnake venom due to its high variation in metalloproteinase composition and biochemical activities. Previous published work has shown that an inverse relationship exists between the presence of the Mojave toxin (a potent neurotoxin) and the presence of hemorrhagic toxins (metalloproteinase) in the venom of this species of rattlesnakes $[15,16]$. Our biochemical data confirm our previous observations that the Mojave rattlesnake venom is highly variable across different geographic locations. In the 
sample population analyzed for this study, we found that the rattlesnake venom derived from a mother and two of her offspring contained strong hemorrhagic activity. These rattlesnakes were captured in El Paso County, TX, USA. Moreover, a rattlesnake containing strong hemorrhagic activity was collected in Maricopa County, AZ, USA. Two other snakes that were collected in Maricopa County, AZ, USA, also had modest hemorrhagic activity, whereas other snakes caught in Texas, New Mexico, and some in Arizona had venoms with weak proteolytic activity and no hemorrhagic activity. Overall, these observations attest to the importance of producing antisera against biochemically different types of Mojave rattlesnake venom.

Rattlesnakes have different types of metalloproteinases in their venom, which have been classified according to their hemorrhagic, fibrinogenolytic and/or fibrinolytic, anticomplement, and a combination of these proteolytic activities $[5,6,23,35,36]$. Fibrinogen, fibrin, casein, and hide powder azure are substrates often used to measure proteolytic activity of crude rattlesnake venom or components of venom. A broad-spectrum proteolytic activity against different substrates is associated with high hemorrhagic activity. In the present study, we assessed metalloproteinase activities in Mojave rattlesnake venom based on its ability to hydrolyze different substrates. We found significant intra-species differences in venom metalloproteinase-related biochemical activity and hemorrhagic activity for C. s. scutulatus rattlesnake. Mojave rattlesnake venoms that were active against hide powder azure correlated with high hemorrhagic activity and had broad activity against all other substrates. Rattlesnake venoms that were active against three of four substrates had modest to weak hemorrhagic activity suggesting that a combination of proteolytic and fibrinogenolytic enzymes are required to induce potent hemorrhagic activity (Table 3). Pretreatment with EDTA, as expected, resulted in a complete loss of metalloproteinase-specific activities, except for fibrinogen, which was hydrolyzed by all rattlesnake venoms even in the presence of EDTA suggesting that all C. s. scutulatus venoms contained esterase and/or serine proteases, enzymes known to hydrolyze fibrinogen in the absence of metals [29, 30].

Other species of the Crotalidae family also show strong intra-species variation. Indeed, we have previously shown that a population of C. m. molossus had up to eleven fibrin hydrolyzing enzymes $[4,5]$ and at least 13 metalloproteinases including Ht-a, Ht-b, Ht-c, Ht-d, Ht-e, Ht-f, Ht-g, VAP1, VAP2, Catroxase, and Atroxases [36]. Unlike all the other comparative biochemical studies previously done, this is the first report to show a genetic basis for differences in metalloproteinase activity of the Mojave rattlesnake. Prior to this study, the molecular mechanisms that give rise to the variability in rattlesnake venom metalloproteinase activity within members of the same rattlesnake species such as $C$. scutulatus scutulatus were not known. Other investigators have postulated that certain environmental factors and/or diet associated with geographical location may modulate the transcription, translation, and/or posttranslational processing of metalloproteinases and contribute to intraspecies variation of rattlesnake biochemical profiles.
Furthermore, this is the first study to categorize Mojave rattlesnake metalloproteinases into four groups based on their DNA homology and biochemical profiles. Indeed, Groups 2, 3, and 4 Mojave rattlesnake metalloproteinase genes have few single nucleotide differences that may encode for minor coding differences at the $\mathrm{N}$-terminal and central regions of the proteinase domain that may likely decrease or nullify the hemorrhagic activities of these three groups of metalloproteinase genes. Interestingly, the C-terminal regions of the metalloproteinase genes are very well conserved among the four groups, which suggests that the presence of additional secondary structural elements contributes to hemorrhagic induction and that they are distinct from the disintegrin domain, a region involved in inhibiting platelet aggregation. Although both subclasses of metalloproteinases may show proteolytic activity towards different substrates, hemorrhagic activity may be imparted by additional structural domains beyond the proteinase domain. Based on the biochemical profile and genomic DNA sequencing data, we believe that Group 1 of Mojave rattlesnake metalloproteinase genes is classified as a P-1A metalloproteinase, whereas other groups are likely classified as P-1B metalloproteinase given their lack or little hemorrhagic activity. Interestingly, none of the groups correlated with geographic location but rather showed a perfect association with their biochemical profiles suggesting that each group displays different patterns of migration across the southwest region of the USA. Interestingly, our results suggest that single nucleotide polymorphisms within the mature proteinase domain may further contribute to secondary structural differences that lead to differences in catalytic activity of metalloproteinases.

In this study, we discovered the presence of an intron that is located within the metal-binding region of the metalloproteinase sequence. Alternative splicing of a large primary transcript has also been proposed as an alternative molecular mechanism that gives rise to differences in metalloproteinase composition and biochemical profiles of rattlesnake venom [7]. This model suggests that a single large primary transcript gives rise to P-I, P-II, and/or P-III metalloproteinases by alternative splicing of a large metalloproteinase primary transcript. The observation in this study that the noncoding regions for the four groups of rattlesnakes showed low homology ( $\sim 67 \%)$ compared to the exon coding regions suggests that possible alternate splicing mechanisms can give rise to different metalloproteinase splice variants leading to differences in metalloproteinase composition of rattlesnake venom of C. s. scutulatus. Indeed, the intron in GP2 was the least similar to the other three groups. Interestingly, while all genomic DNA sequences contained the same consensus splice sites, the DNA homology of the intron region was at least $67 \%$ between GP2 and the other groups of the metalloproteinase genes sequenced in C. s. scutulatus rattlesnakes. Although there is a general consensus that exons are more conserved than introns within the genes of the same family [37], a 67\% nucleotide sequence identity for the intron regions especially for rattlesnakes belonging to the same species is quite low and unexpected.

The untranslated region sequences (UTR) in mRNA transcripts are small intronic regions contained in genes [37] 
that produce secondary RNA structures involved in the regulation of translation [38]. In this study, we demonstrated that the intron regions of C. s. scutulatus contain an unusually low DNA sequence homology, which gives rise to the possibility that different secondary structures generated by introns may modulate transcription of metalloproteinases. Overall, based on our genomic DNA sequences and biochemical data, we believe that a combination of alternative splicing, single nucleotide polymorphisms within the catalytic regions of the proteinase domains, and differences in UTR structures contribute to intra-species variation in metalloproteinase composition and hemorrhagic activity in the Mojave rattlesnake. However, we do recognize that our results do not directly show that alternative splicing contributes to differences in rattlesnake venom composition and metalloproteinase-associated activities, and future in vitro transcription/translation studies are required to conclusively test this hypothesis.

\section{Conclusions}

We identified four groups of C. s. scutulatus rattlesnake venoms with each having distinct biochemical profiles associated with metalloproteinase activity. Rattlesnakes from each venom group had unique genomic nucleotide DNA sequences for the mature metalloproteinase domain and noncoding regions. These findings support the genomic basis underlying diversity in venom metalloproteinase activities. This is the first report on genomic DNA sequences of snake venom metalloproteinases.
Abbreviations
PCR: Polymerase chain reaction
TAME: N-a-p-Tosyl-L-arginine methyl ester
BAEE: N-a-Benzoyl-L-arginine ethyl ester
EDTA: Ethylenediaminetetraacetic acid
cDNA: Complementary DNA
mRNA: Messenger RNA
UTR: Untranslated region.

\section{Conflict of Interests}

The authors of this study do not declare any conflict of interests with the use of Vector NTI, a registered trademark of Life Technologies (Grand Island, NY, USA), during the study.

\section{Acknowledgments}

This study was supported by NIH Grants S06GM08012, RR08124, and GM103554. The authors also acknowledge the technical assistance of Mr. Mario Ramos-Castillo and Ms. Chidem Keskin.

\section{References}

[1] F. S. Markland, "Rattlesnake venom enzymes that interact with components of the hemostatic system," Journal of Toxicology, vol. 2, no. 2, pp. 119-160, 1983.
[2] O. Molina, R. K. Seriel, M. Martinez, M. L. Sierra, A. VarelaRamirez, and E. D. Rael, "Isolation of two hemorrhagic toxins from Crotalus basiliscus basiliscus (mexican west coast rattlesnake) venom and their effect on blood clotting and complement," International Journal of Biochemistry, vol. 22, no. 3, pp. 253-261, 1990.

[3] E. D. Rael, C. S. Lieb, N. Maddux, A. Varela-Ramirez, and J. Perez, "Hemorrhagic and Mojave toxins in the venoms of the offspring of two Mojave rattlesnakes (Crotalus scutulatus scutulatus)," Comparative Biochemistry and Physiology B, vol. 106, no. 3, pp. 595-600, 1993.

[4] E. D. Rael, J. Z. Rivas, T. Chen, N. Maddux, E. Huizar, and C. S. Lieb, "Differences in fibrinolysis and complement inactivation by venom from different northern blacktailed rattlesnakes (Crotalus molossus molossus)," Toxicon, vol. 35, no. 4, pp. 505513, 1997.

[5] T. Chen and E. D. Rael, "Purification of M5, a fibrinolytic proteinase from Crotalus molossus molossus venom that attacks complement," International Journal of Biochemistry and Cell Biology, vol. 29, no. 5, pp. 789-799, 1997.

[6] E. D. Rael, M. Martinez, and O. Molina, "Isolation of a fibrinolytic protease, M4, from venom of Crotalus molossus molossus (Northern blacktail rattlesnake)," Haemostasis, vol. 22, no. 1, pp. 41-49, 1992.

[7] L. A. Hite, L.-G. Jia, J. B. Bjarnason, and J. W. Fox, "cDNA sequences for four snake venom metalloproteinases: structure, classification, and their relationship to mammalian reproductive proteins," Archives of Biochemistry and Biophysics, vol. 308, no. 1, pp. 182-191, 1994.

[8] L. Jia, X. Wang, J. D. Shannon, J. B. Bjarnason, and J. W. Fox, "Function of disintegrin-like/cysteine-rich domains of atrolysin A. Inhibition of platelet aggregation by recombinant protein and peptide antagonists," Journal of Biological Chemistry, vol. 272, no. 20, pp. 13094-13102, 1997.

[9] J. M. Gutiérrez and A. Rucavado, "Snake venom metalloproteinases: their role in the pathogenesis of local tissue damage," Biochimie, vol. 82, no. 9-10, pp. 841-850, 2000.

[10] L. G. Jia, X. Wang, J. D. Shannon, J. B. Bjarnason, and J. W. Fox, "Inhibition of platelet aggregation by the recombinant cysteine-rich domain of the hemorrhagic snake venom metalloproteinase, atrolysin A," Archives of Biochemistry and Biophysics, vol. 373, no. 1, pp. 281-286, 2000.

[11] Q. Zhou, P. Hu, M. R. Ritter et al., "Molecular cloning and functional expression of contortrostatin, a homodimeric disintegrin from southern copperhead snake venom," Archives of Biochemistry and Biophysics, vol. 375, no. 2, pp. 278-288, 2000.

[12] J. B. Bjarnason and J. W. Fox, "Hemorrhagic metalloproteinases from snake venoms," Pharmacology and Therapeutics, vol. 62, no. 3, pp. 325-372, 1994.

[13] K. Shimokawa, L. Jia, X. Wang, and J. W. Fox, "Expression, activation, and processing of the recombinant snake venom metalloproteinase, Pro-atrolysin E," Archives of Biochemistry and Biophysics, vol. 335, no. 2, pp. 283-294, 1996.

[14] M. Anaya, E. D. Rael, C. S. Lieb, J. C. Perez, and R. J. Salo, "Antibody detection of venom protein variation within a population of prairie rattlesnake Crotalus v. viridis," Journal of Herpetology, no. 264, pp. 473-482, 1992.

[15] J. L. Glenn and R. Straight, "Mojave rattlesnake Crotalus scutulatus scutulatus venom: variation in toxicity with geographical origin," Toxicon, vol. 16, no. 1, pp. 81-84, 1978.

[16] J. L. Glenn, R. C. Straight, M. C. Wolfe, and D. L. Hardy, "Geographical variation in Crotalus scutulatus scutulatus (Mojave 
rattlesnake) venom properties," Toxicon, vol. 21, no. 1, pp. 119130,1983 .

[17] L. G. Jia, K. Shimokawa, J. B. Bjarnason, and J. W. Fox, "Snake venom metalloproteinaes: structure, function and relationship to the adams family of proteins," Toxicon, vol. 34, no. 11-12, pp. 1269-1276, 1996.

[18] B. J. Wooldridge, G. Pineda, J. J. Banuelas-Ornelas et al., "Mojave rattlesnakes (Crotalus scutulatus scutulatus) lacking the acidic subunit DNA sequence lack Mojave toxin in their venom," Comparative Biochemistry and Physiology B, vol. 130, no. 2, pp. 169-179, 2001.

[19] W. J. French, W. K. Hayes, S. P. Bush, M. D. Cardwell, J. O. Bader, and E. D. Rael, "Mojave toxin in venom of Crotalus helleri (Southern Pacific Rattlesnake): molecular and geographic characterization," Toxicon, vol. 44, no. 7, pp. 781-791, 2004.

[20] D. J. Massey, J. J. Calvete, E. E. Sánchez et al., "Venom variability and envenoming severity outcomes of the Crotalus scutulatus scutulatus (Mojave rattlesnake) from Southern Arizona," Journal of Proteomics, vol. 75, no. 9, pp. 2576-2587, 2012.

[21] L. A. Hite, J. W. Fox, and J. B. Bjarnason, "A new family of proteinases is defined by several snake venom metalloproteinases," Biological Chemistry Hoppe-Seyler, vol. 373, no. 7, pp. 381-385, 1992.

[22] O. Zhou, B. J. Smith, and M. H. Grossman, "Molecular cloning and expression of catrocollastatin, a snake-venom protein from Crotalus atrox (western diamondback rattlesnake) which inhibits platelet adhesion to collagen," Biochemical Journal, vol. 307, no. 2, pp. 411-417, 1995.

[23] M. Martinez, E. D. Rael, and N. L. Maddux, "Isolation of a hemorrhagic toxin from mojave rattlesnake (Crotalus scutulatus scutulatus) venom," Toxicon, vol. 28, no. 6, pp. 685-694, 1990.

[24] R. A. Martinez, S. Y. Huang, and J. C. Perez, "Antigenic relationships of fractionated western diamondback rattlesnake (Crotalus atrox) hemorrhagic toxins and other rattlesnake venoms as indicated by monoclonal antibodies," Toxicon, vol. 27, no. 2 , pp. 239-245, 1989.

[25] E. D. Rael and L. P. Jones, "Isolation of an anticomplement factor from the venom of the Mojave rattlesnake (Crotalus scutulatus scutulatus)," Toxicon, vol. 21, no. 1, pp. 57-65, 1983.

[26] J. B. Bjarnason and A. T. Tu, "Hemorrhagic toxins from western diamondback rattlesnake (Crotalus atrox) venom: isolation and characterization of five toxins and the role of zinc in hemorrhagic toxin," Biochemistry, vol. 17, no. 16, pp. 3395-3404, 1978.

[27] J. G. Soto, J. C. Perez, M. M. Lopez et al., "Comparative enzymatic study of HPLC-fractionated Crotalus venoms," Comparative Biochemistry and Physiology B, vol. 93, no. 4, pp. 847-855, 1989.

[28] S. Y. Huang, J. C. Perez, E. D. Rael, C. Lieb, M. Martinez, and S. A. Smith, "Variation in the antigenic characteristics of venom from the Mojave rattlesnake (Crotalus scutulatus scutulatus)," Toxicon, vol. 30, no. 4, pp. 387-396, 1992.

[29] M. W. Schwartz and A. L. Bieber, "Characterization of two arginine ester hydrolases from Mojave rattlesnake (Crotalus scutulatus scutulatus) venom," Toxicon, vol. 23, no. 2, pp. 255269, 1985.

[30] A. H. Henschen-Edman, I. Theodor, B. F. P. Edwards, and H. Pirkle, "Crotalase, a fibrinogen-clotting snake venom enzyme: primary structure and evidence for a fibrinogen recognition exosite different from thrombin," Thrombosis and Haemostasis, vol. 81, no. 1, pp. 81-86, 1999.
[31] H. de Araujo and C. Ownby, "Molecular cloning and sequence analysis of cDNAs for metalloproteinases from broad-banded copperhead Agkistrodon contortrix laticinctus," Archives of Biochemistry and Biophysics, vol. 320, no. 1, pp. 141-148, 1995.

[32] Y. Jia, B. A. Cantu, E. E. Sánchez, and J. C. Pérez, "Complementary DNA sequencing and identification of mRNAs from the venomous gland of Agkistrodon piscivorus leucostoma," Toxicon, vol. 51, no. 8, pp. 1457-1466, 2008.

[33] Y. Jia and J. C. Pérez, "Molecular cloning and characterization of cDNAs encoding metalloproteinases from snake venom glands," Toxicon, vol. 55, no. 2-3, pp. 462-469, 2010.

[34] H. E. Van Wart and H. Birkedal-Hansen, "The cysteine switch: a principle of regulation of metalloproteinase activity with potential applicability to the entire matrix metalloproteinase gene family," Proceedings of the National Academy of Sciences of the United States of America, vol. 87, no. 14, pp. 5578-5582, 1990.

[35] L. A. Hite, J. D. Shannon, J. B. Bjarnason, and J. W. Fox, "Sequence of a cDNA clone encoding the zinc metalloproteinase hemorrhagic toxin e from Crotalus atrox: evidence for signal, zymogen, and disintegrin-like structures," Biochemistry, vol. 31, no. 27, pp. 6203-6211, 1992.

[36] J. B. Bjarnason and J. W. Fox, "Hemorrhagic metalloproteinases from snake venoms," Pharmacology and Therapeutics, vol. 62, no. 3, pp. 325-372, 1994.

[37] N. Maeda and O. Smithies, "The evolution of multigene families: human haptoglobin genes," Annual Review of Genetics, vol. 20, pp. 81-108, 1986.

[38] K. Nakashima, I. Nobuhisa, M. Deshimaru et al., "Accelerated evolution in the protein-coding regions is universal in crotalinae snake venom gland phospholipase A2 isozyme genes," Proceedings of the National Academy of Sciences of the United States of America, vol. 92, no. 12, pp. 5605-5609, 1995. 

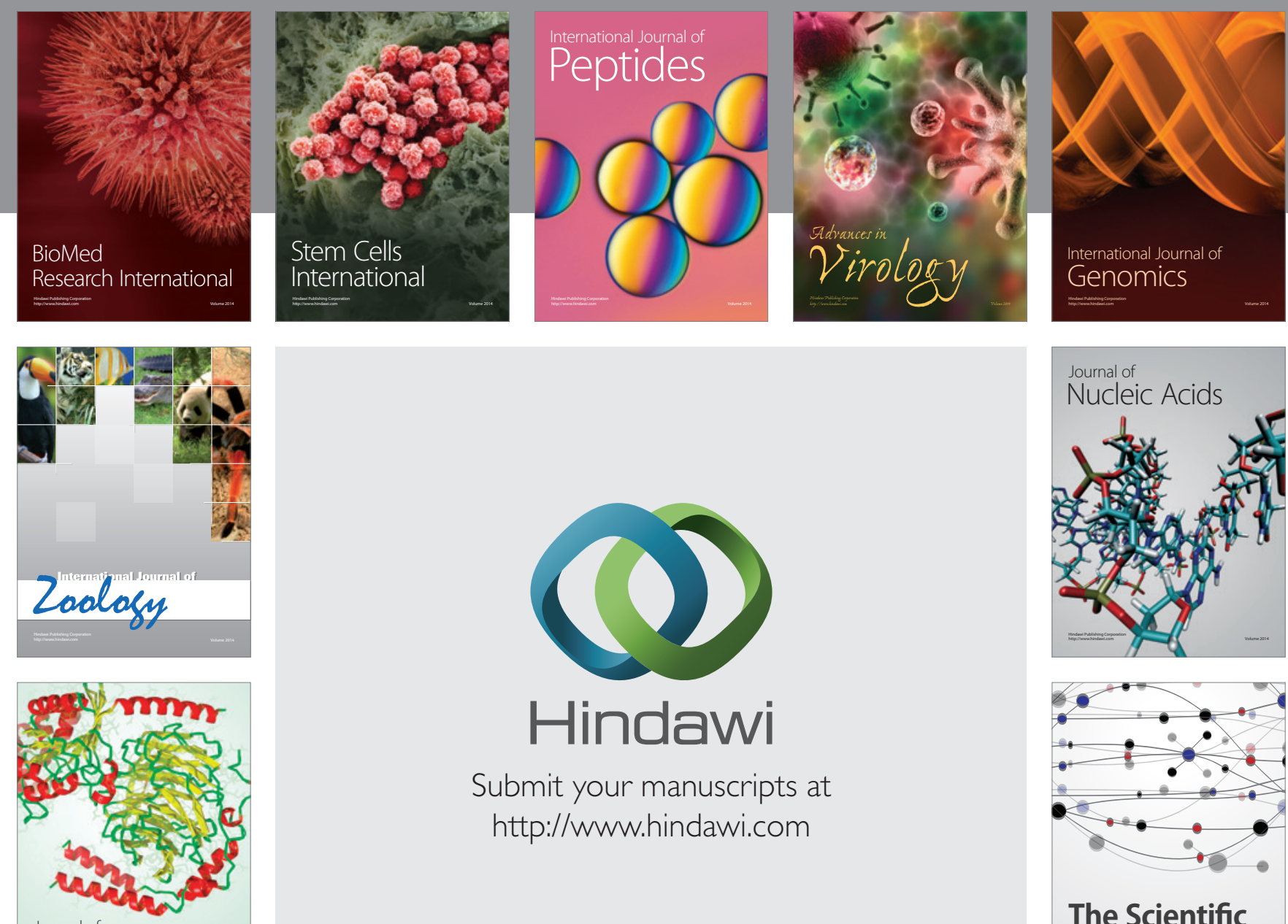

Submit your manuscripts at

http://www.hindawi.com

Journal of
Signal Transduction
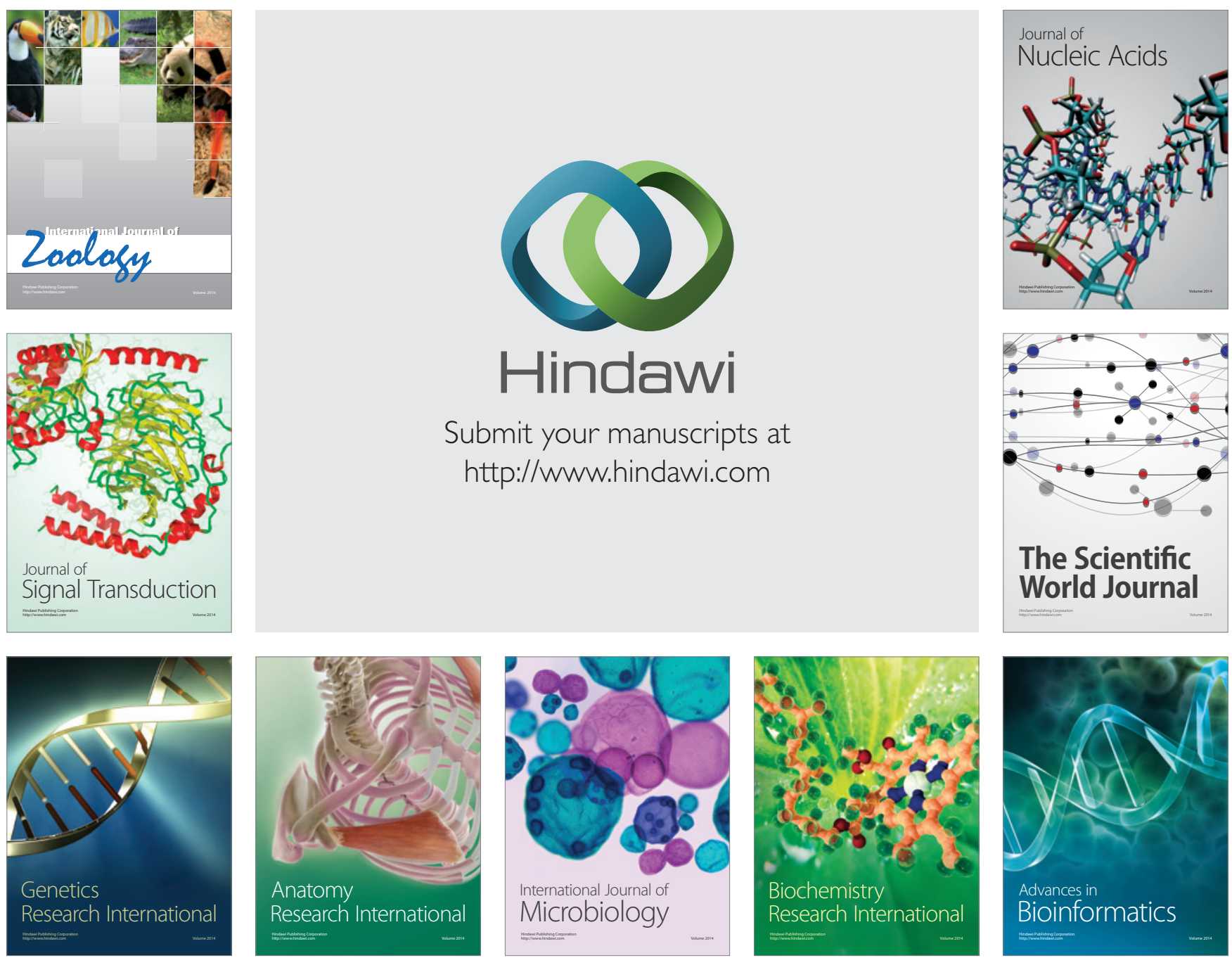

The Scientific World Journal
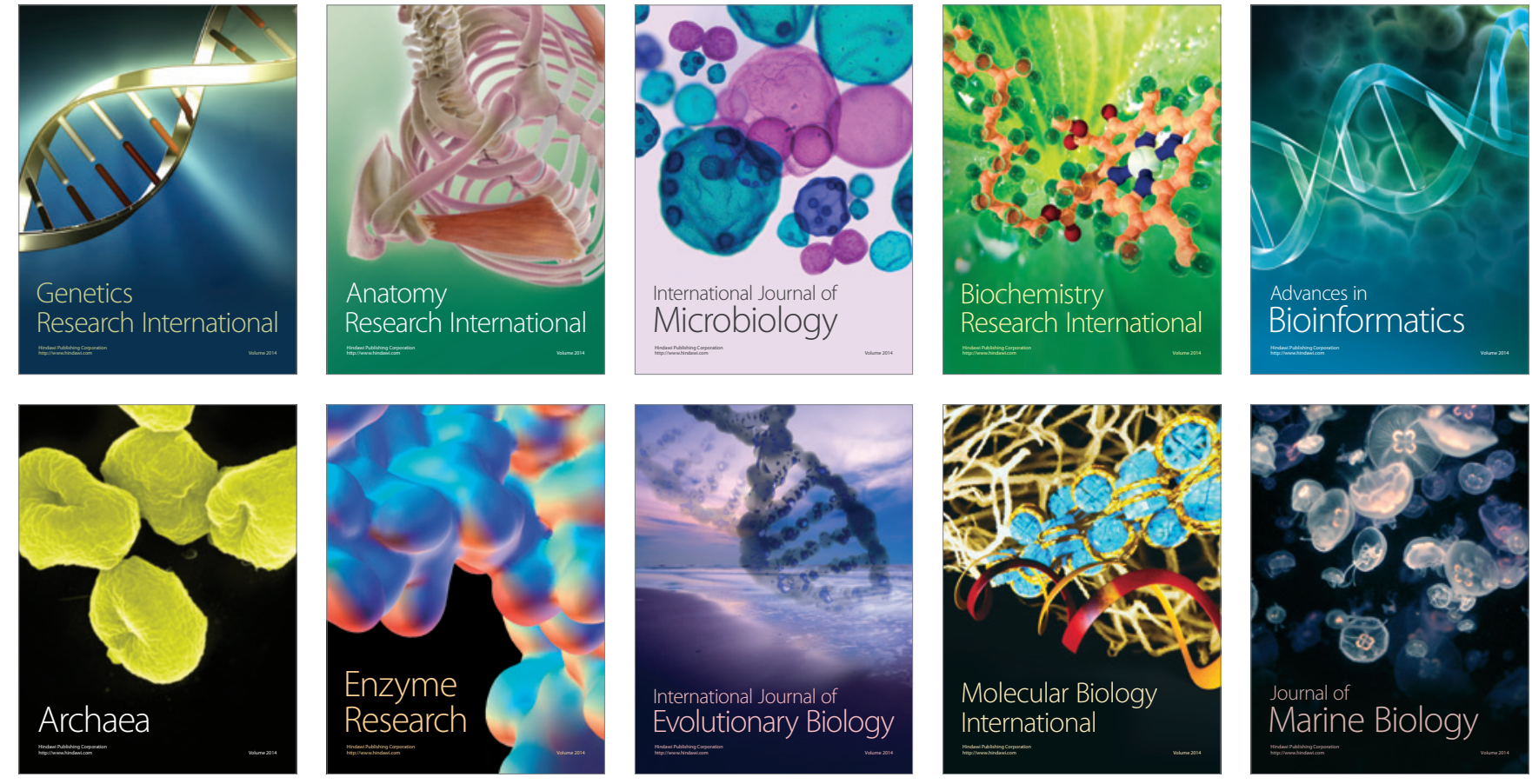